\title{
CASPER CALLS FOR EDUCATION FOR PRACTICE MANAGERS
}

The Campaign for Administrative Standards and Professional Education for Receptionists and Practice Managers (CASPER) is calling for support.

In a statement from Glenys Bridges and Jane Armitage, CASPER says that 'when it comes to the non-clinical aspects of dental care there is a massive black hole in terms of training and ongoing development requirements'

The statement describes dentistry's progress from the Dentist Act 1921 to the past ten years when the curricular framework was introduced to enable members of the dental team to gain registrable qualifications and increase the scope of their contribution to patient care. The statement concedes that the quality of UK clinical dentistry is world class, but says that to consistently achieve an excellent dental experience for patients, a range of quality management skills are required, such as planning services, auditing performance, creating, implementing and evaluating SMART objectives and gathering feedback on clinical and non-clinical aspects of care.

'Without formal education these skills will be absent from dental teams' skill sets, therefore patients' experiences will suffer, says the statement.

CASPER has gathered high profile dental professionals who believe that qualifications for practice mangers and receptionists are the next logical step in the progression of the dental profession and are urging the GDC and CQC to formalise a non-clinical curricular framework.

CASPER invites individuals who support the campaign to email them at casper.campaign@gmail.com with their name and the following statement:

'I agree that the dental profession needs definitive non-clinical educational and CPD standards for dental managers and administrators.'

\section{ADVICE PORTAL LAUNCHED FOR MANAGERS}

First Practice Management (FPM) has launched a dedicated version of its online information portal for dental practice managers. The site offers a breadth of information and resources which have been developed specifically by dental experts.

By signing up to the portal, practice managers can access practical guidance and support with regulatory compliance across areas such as human resources and employment law, health and safety and practice administration. The portal's extensive information and protocols library provides the tools to address issues that are of utmost importance to dental practices such as the Care Quality Commission's (CQC's) standards of quality and safety.

The portal is called Dental Practice Management (DPM) and a subscription includes unlimited access to advice lines along with frequent bulletin updates containing news, articles and notification of upcoming changes to legislation.

www.dentalpracticemanagement.co.uk

\section{BOOK EXPLORES GUM DISEASE-SYSTEMIC HEALTH LINK}

The textbook Periodontal disease and overall health: a clinician's guide was launched recently at Chandos House in London and is available to all dental professionals to download as a PDF from www.colgateprofessional.co.uk.

The launch was attended by a wide range of educators and periodontists.

The publication, supported by an educational grant from Colgate, was edited by Drs Robert J. Genco and Ray C. Williams; their goal to 'present the emerging and compelling evidence that periodontal disease is a risk for several systemic conditions and to look at the role of oral health in contributing to overall health'. The book also seeks to provide the reader with a guide to patient management in which dentistry and medicine work together. 